\title{
Die Welt aus den Fugen
}

$\mathrm{H}$ euschnupfen im Sinne von sommerlichem Schnupfen, Niesanfällen und Augentränen - das war einmal. Im Winter 2003/ 2004 kamen die ersten Patienten um Weihnachten herum mit den typischen Zeichen einer allergischen Konjunktivitis und Rhinitis, in Einzelfällen auch mit Asthma bronchiale, in Behandlung. Pollenregistrierungen für die Stiftung Deutscher Polleninformationsdienst wurden in der Vergangenheit erst ab Februar/März durchgeführt - in den letzten Jahren fraglos zu spät, um den Beginn der Pollensaison zu erfassen. 300 ist die magische Grenze:

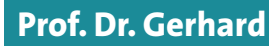

Schultze-Werninghaus

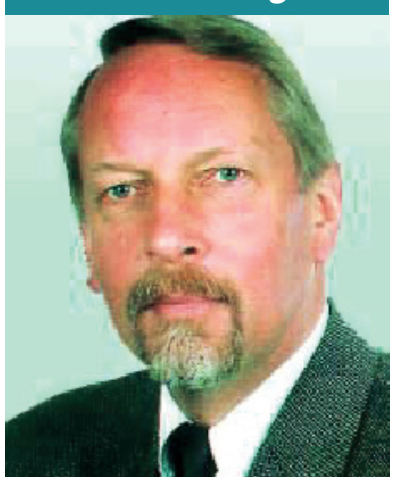

Abteilung für Pneumo-

logie, Allergologie und Schlafmedizin, BG Kliniken Bergmannsheil Universitätsklinik, Bochum

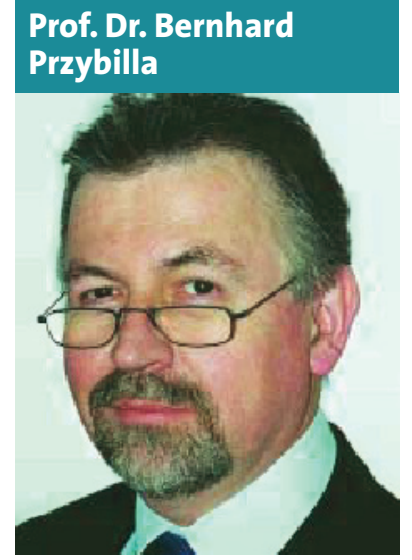

Klinik und Poliklinik für Dermatologie und Allergologie, Klinikum der Ludwig-MaximiliansUniversität, München

dert, Generika als solche zu deklarieren - die Forderung stieß auf scharfe Gegenreaktion - unklar, warum. Patienten mit allergischen Erkrankungen sind durch weitere Maßnahmen des Gesundheitsreformgesetzes verunsichert, nicht zuletzt durch die Frage, welche Medikamente beim Apotheker freiverkäuflich erworben, welche vom behandelnden Arzt verordnet werden sollen. Für den behandelnden Arzt ist unklar, ob er wegen der Zuzahlung des Patienten vorzugsweise Großpackungen verordnen soll, oder aber wie in der Vergangenheit zunächst im Sinne einer Versuchsphase Kleinpak-

den negativen finanziellen Folgen? Derzeit wird vom entsprechenden Bundesit programmiert. Es liegt nahe, dass diese Phänomene zu der Zunahme allergischer Erkrankungen beitragen, nicht zuletzt, da Frühblüher durch zahlreiche Kreuzallergien ganzjährige allergische Reaktionen fördern können - durch orale Allergenzufuhr, wie von Nüssen, Steinund Kernobst, Kiwi u.v.a.

Allergische Patienten sind, nicht zuletzt durch die frühzeitige Beschwerdesaison, von den aktuellen Entwicklungen der Gesundheitsreform besonders betroffen. Leiden sie, nach Auffassung des zuständigen Bundesausschusses, etwa nicht an einer chronischen Krankheit, mit den entsprechen-

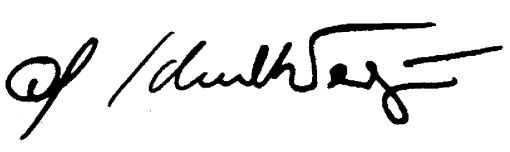

Prof. Dr. G. Schultze-Werninghaus ausschuss die Pollinose offenbar als „Bagatellerkrankung “ eingeschätzt - als ob in Berlin nicht zur Kenntnis genommen würde, dass die allergische Rhinokonjunktivitis international als „major chronic respiratory disease" eingestuft wird, nicht zuletzt in der WHO-unterstützten ARIA-Initiative.

Inwieweit sind antiallergische Generika für die Behandlung geeignet? Welche Risiken durch Generika bestehen, z. B.

kungen. Weitere Unsicherheiten könnten benannt werden.

Fazit: Die allgemeine Verunsicherung ist groß, Einsparungen im Gesundheitswesen sind fraglich. Daher kann zunächst nur der Versuch unternommen werden, für die ca. 30\% von Allergien betroffenen Menschen in der Bevölkerung Regelungen im Gesundheitssystem zu finden, die diesem chronischen Krankheitszustand gerecht werden. Einerseits gibt es Disease Management Programme für Asthma, andererseits ist selbst bei diesem Krankheitsbild fraglich, ob es als chronische Krankheit zu bewerten ist - das kann nicht hingenommen werden.

Allergologisch tätige Kollegen aller bezüglich der Wirksamkeit oder der eventuell unerwünschten Wirkungen von Begleitstoffen? Wie groß ist das Risiko, gefälschten Medikamenten ausgesetzt zu werden? Die DGAI hat gegenüber dem Bundesministerium geforFachrichtungen sind aufgefordert, über die entsprechenden Fachverbände aktiv in die derzeitigen Veränderungen im Gesundheitssystem konstruktive Vorschläge einzubringen.

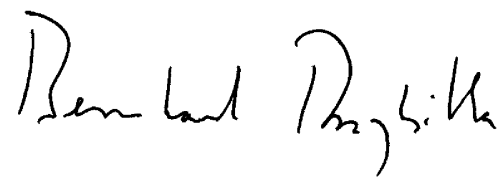

Prof. Dr. B. Przybilla 